\title{
Patient flow simulation modelling - an approach conducive to multi-disciplinary collaboration towards hospital capacity management
}

\author{
M. Mackay ${ }^{a}$, S. Qin ${ }^{\text {b }}$, A. Clissold ${ }^{\text {b }}$, P. Hakendorf ${ }^{\text {c }}$, D. Ben-Tovim ${ }^{\text {c }}$, G McDonnell ${ }^{\text {d }}$ \\ ${ }^{a}$ School of Medicine, Flinders University, South Australia \\ ${ }^{b}$ School of Computer Science, Engineering and Mathematics, Flinders University, South Australia \\ ${ }^{c}$ Flinders Medical Centre, South Australia \\ ${ }^{d}$ University of New South Wales, New South Wales \\ Email: mark.mackay@flinders.edu.au
}

\begin{abstract}
Emergency hospitals continue to face increasing demand and operate at high occupancy levels resulting in more frequent crowding and prolonged waiting. These issues are not expected to abate, but may become more frequent as hospital activity is predicted to increase.

Patient wait and crowding issues are outcomes that can be attributed to multiple causes. It will be important for hospital and health care managers to adopt new tools that will enable them to investigate the complex issue of patient flow through the emergency department and hospital in order to improve patient flow and minimise delays.
\end{abstract}

Simulation has been employed in various industries to achieve process improvement. Although simulation has been employed to investigate patient flow issues in health care its use as a routine management tool is yet to occur. The lack of up take of simulation may be attributed to a variety of factors including confusion about which methodology should be employed and the capacity to accurately model the complex health environment.

There are a variety of approaches to simulation including systems dynamic, discrete event and agent based modelling. Until recently off the shelf software packages enabled the modelling of systems using a single method. AnyLogic (anylogic.com) has been identified as a software package that enables a multi-method approach to be adopted and has been sufficiently proven in its use to warrant investigation as a tool to assist in the creation of simulation models of patient flow. The increased capacity of computers and the reduction in costs of both hardware and software are also no longer a barrier to the implementation of simulation as a means of investigating health care management problems.

The possession of modelling skills alone is insufficient to enable the development of a meaningful simulation patient flow model. Multi-disciplinary collaboration between a range of professionals is also required to enable the capture of the necessary information required to describe the hospital processes, analyse the data, create the and validate the model.

The aim of this project is to create an accurate simulation model of patient flow that accurately describes the progression of patients through the emergency department of a large teaching hospital into general medicine wards and then discharge. This paper describes the development of a concept for simulation modeling of the service and describes an initial model created by a multi-disciplinary team that will be used to investigate scenarios and strategies for improving patient flow through the hospital and reducing waiting times in the emergency department. The visual and quantitative outcome of this study will enable health care professionals and managers to understand the impact of their decisions on the hospital's operation efficiency as well as patient experiences.

Keywords: Patient flow, simulation modelling, hospital capacity management, multi-disciplinary collaboration 


\section{INTRODUCTION}

Australian public hospitals have been under pressure to meet the increasing inpatient care demand as evidenced by the increased public reporting of crowding and delays in admission, and concerns over the cost of the provision of care. Indeed, the demand on Australian Emergency Departments (ED) has been increasing at an annual average of 3.2\% over the period 2006-07 to 2010-11 (AIHW, 2012). Similarly, patient separations (or discharges) increased over the same period by $16.7 \%$ (13.3\% in public acute hospitals and $21.5 \%$ in private hospitals), which represents an annual average increase of $3.9 \%$ (AIHW, 2012).

Like activity levels, expenditure on hospitals has also been increasing, with an annual average growth of $5.0 \%$ after adjusting for inflation for the period 2004-05 to 2009-10 (AIHW, 2011). The combined effect of increasing costs and increasing activity has seen the plight of patients, emergency departments and inpatient services receive constant political and media attention given the subject a high public profile.

The increased occurrence of crowding and waiting is not due to a single cause, but rather a complex mix of population growth and ageing, the rising incidence of chronic diseases associated with modern life and the ability to treat such conditions for a prolonged period, as well as the constraints of limited infrastructure and resources. The pressure on acute care public hospitals is not expected to abate in the future due to the continued ageing of the population and the greater use of public hospitals by older people (FitzGerald et al., 2012).

Aside from the congestion and prolonged periods of waiting, the consequences of operating under duress are potentially accompanied by a reduction in quality of care (Sprivulis et al., 2006). Additionally, it is recognised that staff stress increases during periods of high workloads (Virtanen, Batty, Pentti, Vahtera, et al., 2010).

Although Bagust, Place and Posnett (1999) warned of the consequences of operating at high occupancy levels, the trend for many metropolitan-based hospitals, and also some non-metropolitan-based hospitals, has nevertheless been to operate at higher levels of occupancy and for longer periods of time than in the past. The Australasian College of Emergency Medicine (ACEM) reported (2004) queueing theory indicated that maximum efficiency was achieved when systems operated at 85\% capacity. ACEM (2004) reported that most acute care hospitals were operating at $90-95+\%$ occupancy and therefore in crisis mode frequently and did not have a surge or buffer capacity.

The average $15 \%$ vacancy rate was intended to provide a buffer against the variation in rates of admission of emergency patients. Farmer and Emami (1990) refer to the efficiency factor as opposed to a buffer, but did not specify whether an ideal exists, other than the planning for the required number of beds could be improved. Buffers are used widely in most industrial applications, e.g., telecommunications, to avoid overloading systems, but seem to have been abandoned in the management of hospitals in favour of the pursuit of other goals, such as cost. While the notion of the $85 \%$ level of occupancy may have been brought into question in recent times (Bain, Taylor, McDonnell and Georgiou, 2010) there is nevertheless need for the introduction of improved means of examining hospital systems in order to improve cost and patient care outcomes.

\section{ROLE OF SIMULATION IN LOOKING AT PATIENT FLOW ISSUES}

Health Care data analysis has been traditionally undertaken with statistical techniques and less frequently mathematical techniques in order to report on, understand, improve and forecast issues relating to the management of the health care system. Patterns of activity have been analysed (for example, see Harrison, Zeitz, Adams and Mackay, 2013), but cannot provide a complete picture of the connectedness of the issues facing health care managers.

Simulation provides an opportunity to examine complex systems and can avoid the difficulty of attempting to develop a complex mathematical solution (Law and Kelton, 1991). Health systems are known to be complex and thus it is not surprising that it has been applied to the study of hospital bed occupancy problems previously. For example, simulation methods have been used to study various hospital and health system related issues by Huang (1998), Altinel and Ulaş (1996), El-Darzi, Vasilakis, Chaussalet and Millard (1998), Bagust, Place and Posnett (1999), Lattimer, Brailsford, Turnbull, et al. (2004), Cochran and Bharti (2006), and Taheri et al. (2012). 


\section{CHALLENGES OF PATIENT FLOW SIMULATION}

The volume of literature relating to hospital bed management and patient flow issues has continued to increase and has taken place in various countries, indicating the pervasiveness of hospital bed management and patient flow problems. The application of simulation to improve health care systems, however, has not been without its challenges. For example, there is not a single most effective approach to simulation modelling. Gunal (2012) describes the various modelling approaches. However, for health care managers, the decision to apply what is often to be considered a novel approach to aiding investigation in the health care setting is not aided by the multitude of modelling approaches.

Other issues include insufficiency of data (Lattimer et al., 2004; Cochran and Bharti, 2006), system complexity (Isken and Rajagopalan, 2002), a lack of evaluation of simulation in health care (Fone et al., 2003) and difficulties in generalisability. Additionally, the application of modelling techniques to health care management problems can be seen as a two-streamed process: one where the intended outcome is the development and trialing of methodology, and the other, the reporting of application to real-life situations. Furthermore, the continued reliance upon simple, but poor key performance indicators, such as the average length of stay (Harrison and Millard, 1991), may not help managers gain the insights necessary to improve decision-making and to seek out simulation as a means to improve bed management and patient flow. It is then perhaps not surprising that simulation modelling has not been adopted in the health sector despite the allure of potential improvement in decision-making and outcomes.

\section{THE IMPORTANCE OF COLLABORATION}

Although the application programming interface (API) of modern simulation modelling software has improved considerably in recent years, the need for some level of programming or data analysis skills or both cannot be eliminated completely. While the ability to create a patient flow simulation model of a hospital function can be undertaken by computer and/or data scientists, the ability to create a meaningful model requires the gathering of qualitative and quantitative information. The qualitative information regarding hospital processes is likely to be held by domain experts, namely the medical professionals and other health care staff involved in delivery of patient services. Past experience has shown that analysis or model development that is not undertaken with a multi-disciplinary team on a collaborative basis is less likely to gain acceptance and therefore adoption by health care managers. A particular focus of this work is the inclusion of a collaborative approach to ensure that the modelling outcomes are understood and used. Collaboration in simulation projects has been previously advocated (Rouwette, Korzilius, Vennix, and Jacobs, 2010; Morrison, Rudolph and Carroll, 2013). It is anticipated that the development of the model based upon multi-disciplinary collaboration will help the modellers to more readily communicate implications about changing bed allocations and patient flow in a meaningful manner to other decision-makers within the hospital.

\section{THE SIMULATION PROJECT CONCEPT}

\subsection{The Aim}

The aim of this study is to demonstrate that simulation can be used to improve understand and aid decisionmaking in relation to patient flow and bed management issues. Furthermore, it is intended demonstrate that the creation and engagement of an effective multi-disciplinary collaborative team is helpful in the delivery of the simulation analysis.

\subsection{The Project Hospital Context}

The site for the project is a large public teaching hospital based in the metropolitan city of Adelaide, South Australia. The hospital is a tertiary hospital and currently operates with more than 500 beds. It provides outpatient, emergency department and acute inpatient care services for a population of around 300,000 people. Common problems include severe overcrowding in the emergency department, that has become almost a daily occurrence in recent years, delays in admitting emergency patients and cancellation of booked elective work due to a lack of inpatient beds. Like many such institutions, it is under considerable strain due to sustained increases in demands for care that are not immediately matched by increases in available resources.

There is a need to determine the most efficient ways to improve patient flow and reduce over-crowding events without compromising patient care or staff workloads. The proposed simulation project enables alternative hospital system scenarios to be considered prior to introduction. 


\subsection{Stages of the Simulation}

The project concept can be broken into five stages: 1 . Assembly of the project team, 2. Confirmation of the need, 3. Choice of simulation package, 4. Initial simulation model, and 5. Application to real world problems. Acceptance of the results will represent the success of the project.

\section{Assembly of the Project Team}

Mintzberg (2012) classifies hospitals as professional organisations meaning that many workers hold knowledge. Thus, to access the wide range of knowledge about the hospital operations, and in particular the descriptive knowledge, a collaboration of a group of multi-disciplinary professionals was established to develop a model to assist with the identification of patient flow issues and solutions.

\section{Confirmation of the Need}

Hospital staff that were members of the multi-disciplinary research team confirmed the need to investigate particular aspects relating to patient flow and bed occupancy. The team also refined the nature of the problems to be investigated and confirmed that data were available to enable construction of the simulation model.

\section{Choice of Simulation Package}

Gunal (2012) identified that there are a variety of approaches to hospital model simulation, namely discrete event (DES), systems dynamics and agent-based. Each method serves a particular range of abstraction levels. System dynamics operates at high abstraction level and is mostly used for strategic modeling. Discrete event modeling with the underlying process-centric approach supports medium and medium-low abstraction, where a sequence of operations being performed across entities are examined. Agent based models can vary from very detailed where agents represent passive or active individuals to highly abstract where agent are competing or collaborating organisations.

The choice of method should be based on the system being modeled and the purpose of the modeling. A complex dynamic system needs to be modeled using different methods, i.e., a hybrid or multi-method model is required. Multi-method modeling was not easily done until the availability of AnyLogic (Borshchev \& Filippov, 2004), which enables the inclusion of DES, systems dynamics and agent based approaches in the one model.

\section{Initial Simulation Model}

The patient flow and occupancy problem focused on the movement of patients from the Emergency Department to General Medicine. Patients who are admitted to General Medicine are initially admitted to a short stay ward (AAU) and will be transferred to longer stay in General Medicine if required. Data relating to the patient arrivals at the emergency department (number, day of week and time of day,) and patient transfers (ward, date, time of day) and discharge (day of week) was accessed.

An initial DES model of the process was constructed and is illustrated in Figure 1. 


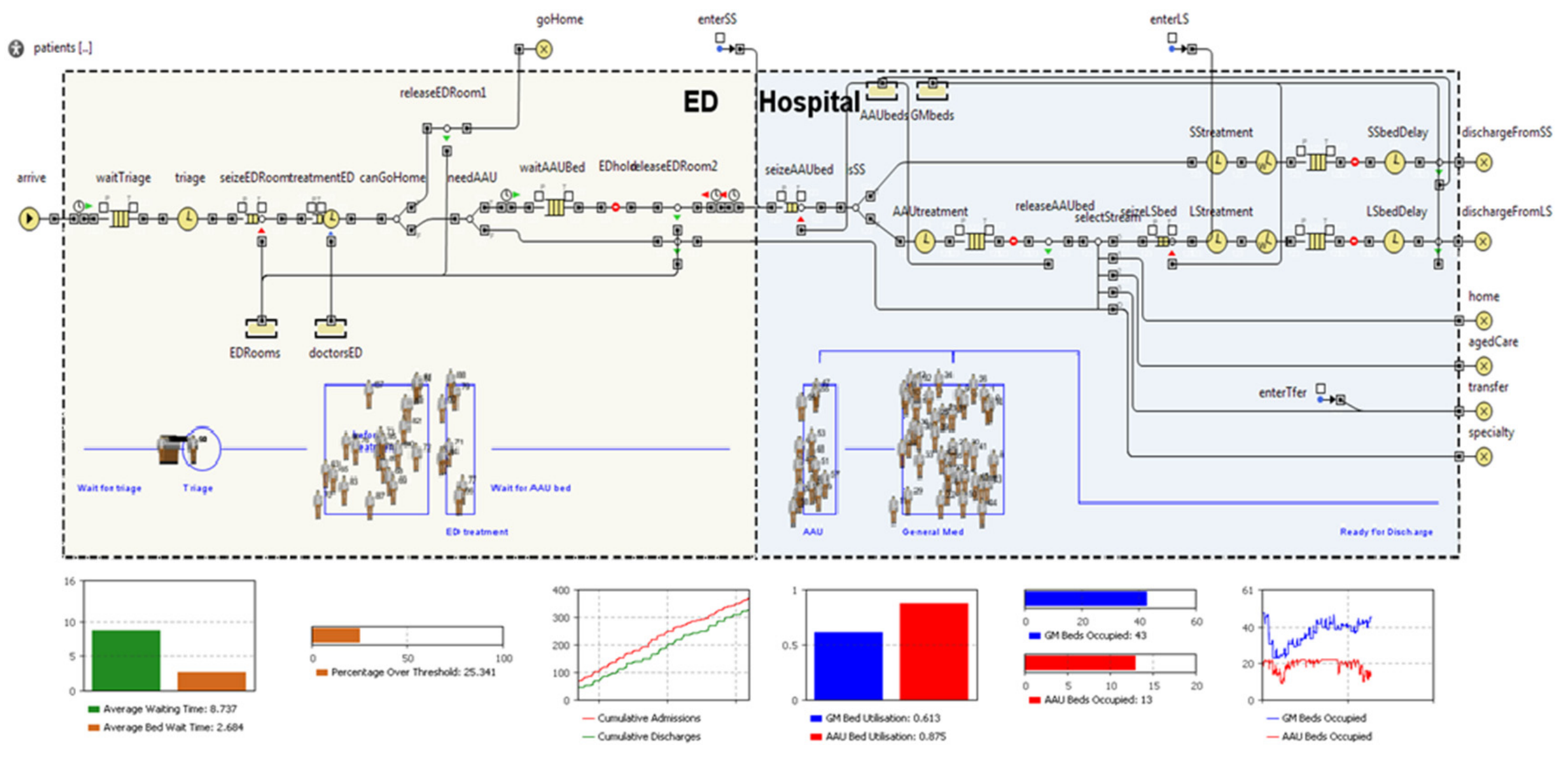

Figure 1: Initial simulation model design for the process of arrival at the Emergency Department, admission to General Medicine, and discharge. 
A simple system dynamics model as shown in Figure 2 is included to capture the health condition of each patient, which can be used for prioritization of ED treatment and bed allocation.

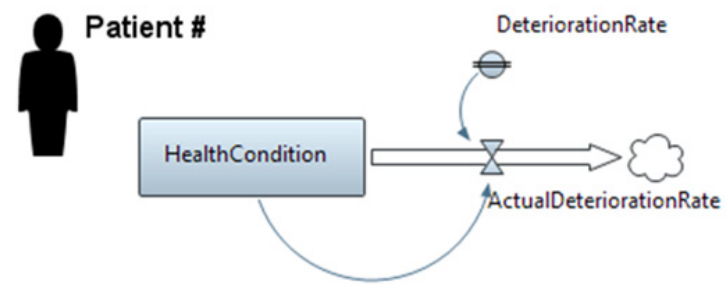

Figure 2: System dynamics model of a patient condition included within the hospital flow model.

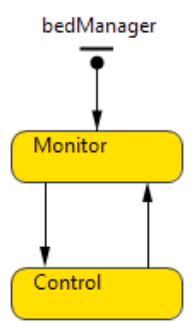

Figure 3: Bed manager modelled as an agent.

The flow of patients is described using discrete event simulation, as there is little advantage in attempting to model the patients as agents in relation to the objectives of this project. The role undertaken by the bed manager, however, can be modeled as an agent, shown in Figure 3. A bed manager will take control action, such as transferring more patients to another hospital or cancel scheduled surgery, if the queue size, ED waiting time and the ward occupancy level reach certain level. System dynamic models within agents may add a quality dimension to the patient flow model and will also be investigated. Other factors, such as the effect of stress on doctors and nurses as occupancy levels increase, may be incorporated into the model using additional agents.

At the time of writing, the research team members had initially reviewed the model and future iterations were being constructed to improve the model's representation of actual hospital processes and enable testing against particular research questions. Additional work involved in the model construction will also include validation against historic hospital data and the inclusion of system dynamics and agent based model components as required.

\section{Application to Real World Problems}

Following iteration of the model and validation that the model replicates past hospital history, the model will be used to investigate, among other things:

- Waiting time and Bed occupancy implications arising from changed bed use

- Waiting time and Bed occupancy implications arising from altering work practices

- Implications of time scale used to track patient movement, and

- Implications of human factors, such as interactions between clinicians and managers, in relation to bed management issues.

This work may require the addition of new modules to the simulation model.

\section{Conclusion}

Simulation modelling has been previously used to investigate health care problems, including patient flow and occupancy related problems. This project endeavours to incorporate a multi-model approach to the development of the simulation model, overcome data and validation issues identified by other authors (e.g., Lattimer et al., 2004; Fone et al., 2003) and ensure that the process is driven by a multi-disciplinary collaboration and multimethod approach to help ensure the creation of robust model that can be applied for considering real world problems by the hospital management. To date, the project team has developed an initial simulation model capable of being used to investigate a range of real-world problems.

\section{References}

ACEM (Australasian College for Emergency Medicine). Access block and over- crowding in emergency departments. April 2004. http://www.acem.org.au/media/Access_Block_2004.pdf (accessed Jul 2013).

AIHW (Australian Institute of Health and Welfare), (2011). Health expenditure Australia 2009-10. Health and welfare expenditure series no. 46. Cat. no. HWE 55. Canberra: AIHW.

AIHW (Australian Institute of Health and Welfare), (2012). Australia's hospitals 2010-11, at a glance. Health services series no. 44. Cat. no. HSE 118. Canberra: AIHW. 
Altinel İK and Ulaş E (1996). Simulation modeling for emergency bed requirement planning. Annals of Operations Research, 67: 183-210.

AnyLogic, http://www.anylogic.com

Bagust A, Place M, Ponsett JW. Dynamics of bed use in accommodating emergency admissions: stochastic simulation model. BMJ 1999; 319:155-158.

Bain, C.A., P.G. Taylor, G. McDonnell and A.Georgiou, Myths of ideal hospital occupancy, Med J Aust 2010; 192 (1): 42-43.

Borshchev, A and A. Filippov (2004). From System Dynamics and Discrete Event to Practical Agent Based Modeling: Reasons, Techniques, Tools. In proceeding of: The 22nd International Conference of the System Dynamics Society, July 25 - 29, 2004, Oxford, England.Cochran JK and Bharti A (2006). Stochastic bed balancing of an obstetrics hospital. Health Care Management Science, Vol 9(1), pp 31-45.

El-Darzi E, Vasilakis C, Chaussalet T and Millard PH (1998). A simulation modelling approach to evaluating length of stay, occupancy, emptiness and bed blocking in a hospital geriatric department. Health Care Management Science, 1: 143-149.

Farmer RDT and Emami J (1990). Models for forecasting hospital bed requirements in the acute sector. Journal of Epidemiology and Community Health, 44, 307-312.

FitzGerald, G., S.Toloo, J. Rego, J.Ting, P.Aitken and V.Tippett. (2012). Demand for public hospital emergency department services in Australia: 2000-2001 to 2009-2010. Emerg Med Australas, 24(1), 72-78.

Fone, D, Hollinghurst, S, Temple, M, Round, A, Lester, N, Weightman, A, Roberts, K, Coyle, E, Bevan, G and Palmer, S (2003). Systematic Review of the use and value of computer simulation modelling in population health and health care delivery. Journal of Public Health Medicine, 25 (4): 325-335.

Gunal MM (2012). A guide for building hospital simulation models. Health Systems (1), 17-25.

Harrison GW, Zeitz K, Adams R and Mackay M (2013). Does hospital occupancy impact discharge rates? Australian Health Review, http://dx.doi.org/10.1071/AH12012

Harrison GW and Millard PH (1991). Balancing acute and long-term care: the mathematics of throughput in departments of geriatric medicine. Methods of Information in Medicine, 30(3): 221-228.

Huang X (1998). Decision making support in reshaping hospital medical services. Health Care Management Science, 1: 165-173.

Isken MW and Rajagopalan B (2002). Data mining to Support simulation modelling of patient flow in hospitals. Journal of Medical Systems, 29(2): 179-196.

Lattimer V, Brailsford S, Turnbull J, Tarnaras P, Smith H, George S, Gerard K and Maslin-Prothero S (2004). Reviewing emergency care systems I: insights from system dynamics modelling. Emergency Medicine Journal, 21(6): 685-91.

Law AM and Kelton WD (1991). Simulation Modeling \& Analysis. 2nd Ed., McGraw Hill, Inc., New York (USA).

Mintzberg H 2012, 'Managing the myths of health care', World Hospitals and Health Services, vol. 48 (3), pp 47 http://www.mintzberg.org/sites/default/files/managing the myths_of health_care.pdf (accessed 20 July 2013).

Morrison, B, Rudolph, JW, and Carroll, JS, (2013). Dynamic modeling as a multidiscipline collaborative journey. System Dynamics Review, vol 29 (1): 4-25

Richardson DB (2006) Increase in patient mortality at 10 days associated with emergency department overcrowding Med J Aust 184 (5):213

Rouwette EAJA, Korzilius, H, Vennix, JAM, and Jacobs, E (2010). Modeling as persuasion: the impact of group model building on attitudes and behavior. System Dynamics Review DOI: 10.1002/sdr.441

Sprivulis, P.C., J.A. Da Silva, I.G. Jacobs, G.A. Jelinek and A.R.L. Frazer. (2006) The association between hospital overcrowding and mortality among patients admitted via Western Australian emergency departments. Med Overcoming the barriers: A qualitative study of simulation adoption in the NHS

Taheri, J, Gellad, Z, Burchfield, D and Cooper, K (2012). A simulation study to reduce nurse overtime and improve patient flow time at a hospital endoscopy unit. Proceedings of the 2012 Winter Simulation Conference C. Laroque, J. Himmelspach, R. Pasupathy, O. Rose, and A. M. Uhrmacher, eds.

Virtanen M, Batty GD, Pentti J, Vahtera J, Oksanen T, Tuisku K, Salo P, Terho K, Ahola K, Elovainio M, Kivimäki M (2010). Patient overcrowding in hospital wards as a predictor of diagnosis-specific mental disorders among staff: a 2-year prospective cohort study. The Journal of Clinical Psychiatry, 71(10):13081312. 